\title{
Unexplained severe lactic acidosis in emergency medicine
}

\section{Ole Erik Ulvin ${ }^{* 1}$ and Erik Waage Nielsen ${ }^{2}$}

\author{
${ }^{1}$ Department of Anesthesiology, Nordland Hospital, Bodo, Norway \\ ${ }^{2}$ Department of Anesthesiology, Nordland Hospital, Bodo, Norway. Institute of Clinical Medicine, University of \\ Tromso, Norway. Faculty of Professional Studies, University of Nordland, Bodo, Norway
}

${ }^{*}$ Corresponding author: Ole Erik Ulvin, Department of Anesthesiology, Nordland Hospital, Bodo, Norway; E-mail: ole.erik.ulvin@gmail.com

Citation: Ole Erik Ulvin, Erik Waage Nielsen (2013) Unexplained severe lactic acidosis in emergency medicine. J Case Rep Stud 1(2): 202. doi: 10.15744/2348-9820.1.202

Received Date: September 03, 2013 Accepted Date: October 15, 2013 Published Date: October 20, 2013

\begin{abstract}
Case report A 49-year-old previously healthy man was admitted to the ICU after cardiac arrest following a short history with headache, blurred speech and reduced consciousness. After cardiopulmonary resuscitation perfusion rhythm was regained, but the patient didn 't regain consciousness. The arterial blood gas analysis at the ICU revealed a severe metabolic acidosis with $\mathrm{pH}$ at 6.86 and lactate levels of $16 \mathrm{mmol} / \mathrm{L}$. The white blood cells count was also markedly increased $\left(312 * 10^{9} / \mathrm{L}\right)$, and blood smear showed immature cells indicating acute leukemia. The severe metabolic acidosis, at first thought to be due to systemic hypoperfusion, did not improve in spite of fluid and vasopressor resuscitation. A CT scan of the head performed the next day, revealed massive cerebellar haemorrhage, edema in both hemispheres and signs of anoxic brain damage. Immunophenotyping of peripheral blood was consistent with Acute Myeloid Leukemia (AML).

Discussion Lactic acidosis is a common cause of metabolic acidosis at the ICU. Type A is most common and caused by hypoperfusion or hypoxia, whilst type B has other causes including use of the antidiabetic drug metformin and hematological malignancies. The latter should be considered when presented to persistent lactic acidosis after adequate systemic perfusion has been reestablished.
\end{abstract}

\section{Introduction}

Lactic acidosis in the ICU is most often seen in relation to systemic hypoperfusion or hypoxia. This case illustrates severe metabolic acidosis with markedly elevated lactate levels following cardiac arrest on another, and far more uncommon, basis.

\section{Case report}

A 49-year-old previously healthy man went to see his physician after about one week with symptoms of the flu including high fever, and was prescribed antibiotics. Two days later his condition dramatically worsened. He had headache, blurred speech and reduced consciousness, and eventually cardiac arrest. His relatives started cardiopulmonary resuscitation, and paramedics arrived after about ten minutes. The air ambulance helicopter arrived after another ten minutes. The first registrated cardiac rhythm was asystole. Perfusion rhythm was regained after a total resuscitation time of 30 minutes. The patient did not regain consciousness. He was intubated and received supportive ventilation during flight to the nearest secondary level of care hospital.

At the time of arrival at the Intensive Care Unit, the patient had atrial fibrillation and his skin was cool and clammy.
His systolic blood pressure was around $60 \mathrm{mmHg}$. He was unconscious with a Glasgow Coma Scale score of 3, and had bilateral fixed dilated pupils. The patient was considered to be too unstable to be transported to the radiology department for a CT scan of the head. ECG showed ST-depressions in the precordial leads. Bedside echocardiography at the ICU revealed a well-contracting left ventricle, although with signs of septal and apical hypokinesia. Thrombolysis on vital indication was considered, but not conducted. A chest $\mathrm{x}$-ray showed diffuse infiltrates bilaterally.

The arterial blood gas analysis at the time of admission revealed a severe metabolic acidosis with $\mathrm{pH}$ at $6.86, \mathrm{pCO}_{2}$ $5.3 \mathrm{kPa}, \mathrm{pO}_{2} 13.4 \mathrm{kPa}, \mathrm{HCO}_{3}-7 \mathrm{mmol} / \mathrm{L}$, Base Excess -24 $\mathrm{mmol} / \mathrm{L}$ and lactate levels of $16 \mathrm{mmol} / \mathrm{L}$. Further laboratory tests showed $\mathrm{Hb} 9.5 \mathrm{~g} / \mathrm{dL}$, INR 1.5, APTT $52 \mathrm{~s}$, thrombocytes $62 * 10^{9} / \mathrm{L}$, fibrinogen $1.6 \mathrm{~g} / \mathrm{L}$, d-dimer $>4 \mathrm{mg} / \mathrm{L}$, ASAT 3085 $\mathrm{U} / \mathrm{L}, \mathrm{ALAT} 1450 \mathrm{U} / \mathrm{L}$, creatinine $420 \mu \mathrm{mol} / \mathrm{L}$ and troponin I at $160 \mathrm{ng} / \mathrm{L}$. The patient's white blood cells count was markedly increased $\left(312 * 10^{9} / \mathrm{L}\right)$. Blood smear showed immature cells indicating acute leukemia, and samples for cytogenetics and immunophenotyping were therefore taken. As leukemia was highly suspected, solumedrol and hydroxyurea were given in agreement with the hematologists. The elevated levels of lactate dehydrogenase $(2310 \mathrm{U} / \mathrm{L})$ combined with hyperphosphatemia and hyperurikemia implied the presence of tumor lysis syndrome; hence allopurinol was given. 
Due to persistent unconsciousness after cardiac arrest, therapeutic hypothermia was implemented for neuroprotection. Amiodarone was given due to rapid atrial fibrillation, and after a couple of hours sinus rhythm was regained. Mean arterial pressure was still 50-60 $\mathrm{mmHg}$ for the first 12 hours at the ICU, but increased eventually to around $70 \mathrm{mmHg}$. It was unknown why the severe metabolic acidosis, at first thought to be due to systemic hypoperfusion, did not improve.

Given the flu-like medical history, meningitis was also suspected and intravenous antibiotic treatment with Penicillin and Ceftriaxon was therefore initiated.

The patient remained anuric after admission to the ICU (total diuresis of $100 \mathrm{ml}$ during the first 12 hours), and in order to try to dialyse acid, phosphate and urate, PRISMA- treatment was started. A bedside colonoscopy to see if bowel ischemia was responsible for the high lactate levels, showed, however, normal and well-circulated intestinal mucosa.

The severe metabolic acidosis sustained in spite of infusion of more than 101 of Ringer Acetate during the first 12 hours at the ICU (approximately $800 \mathrm{ml} /$ hour), in addition to vasoactive medication (norepinephrine $0.25 \mathrm{ug} / \mathrm{kg} / \mathrm{min}$ and epinephrine $0.4 \mathrm{ug} / \mathrm{kg} / \mathrm{min}$ ). The lactate levels continued to rise, peaking at a maxium value of $24 \mathrm{mmol} / \mathrm{L}$ within the same period of time. Intravenous buffer (Tribonate and sodium bicarbonate) did not reduce the acidosis. Although the patient was hyperventilated with $\mathrm{pCO}_{2}$ values at approximately 3.5 $\mathrm{kPa}$, the $\mathrm{pH}$ remained severely low (around 6.9). The cause for this massive lactic acidosis was not identified.

A CT scan of the head taken the day after admission to the ICU, revealed massive cerebellar haemorrhage, edema in both hemispheres and signs of anoxic brain damage. Transcranial Doppler examination demonstrated increased intracranial pressure, and some flow in intracerebral vessels. Neurosurgical expertise considered further treatment futile.

Immunophenotyping of peripheral blood was consistent with Acute Myeloid Leukemia (AML).

Based on the recent findings, withdrawal of active treatment was decided by an interdisciplinary team of doctors and in consultation with the patient's relatives, and the patient died shortly after.

\section{Discussion}

Lactic acidosis is a common cause of metabolic acidosis with increased anion gap amongst patients at the intensive care unit. In 1976, Cohen and Woods presented a subdivision of lactic acidosis into type A and B [1], making type A represent overproduction of lactate due to tissue hypoperfusion like in sepsis or shock, or from tissue hypoxia. Type B lactic acidosis, on the other hand, is characterized by elevated lactate levels from other causes. An example is metformin induced lactic acidosis, which is not seldom seen in the ICU. The antidiabetic agent metformin may trigger lactic acidosis presumably by inhibiting gluconeogenesis and lowering both hepatic uptake and metabolism of lactate to glucose[2]. Another example is deficiency of vitamin B1 (thiamine), an important cofactor in the pyruvat-dehydrogenase complex which is essential to normal aerobic cell metabolism. In addition to this, reversetranscriptase inhibitors used in treatment of HIV and a few congenital diseases are also associated with type B lactic acidosis. Finally, hematological malignancies can give type $\mathrm{B}$ lactic acidosis. The term malignant lactic acidosis is defined as $\mathrm{pH}$ value $\leq 7.37$ together with lactate levels $\geq 5 \mathrm{mmol} / \mathrm{L}$ in association with a tumour [3]. Several explanatory models exist regarding the link between elevated lactate levels and neoplasms. Rapidly growing tumours may outgrow their blood supply, thereby creating a hypoxic environment which will favour anaerobic glycolysis causing lactate formation. It has also been demonstrated that some tumour cells overexpress the glycolytic enzyme hexokinase type II, leading to metabolic imbalance with increased anaerobic glycolysis in preference to gluconeogenesis [3,4]. Leukemic microembolies causing tissue hypoxia and decreased hepatic clearance of lactate due to hepatic leukemic infiltrates, are also possible theories for this phenomenon [5]. We now hold the leukemia mainly responsible for the overwhelming type B lactic acidosis our patient suffered from.

Since severe lactic acidosis on the basis of malignant neoplasms is seen very infrequently compared to the far more common lactic acidosis following hypoperfusion, one might risk a delay in both diagnosis and potentially life saving treatment when facing malignant lactic acidosis. De Raes and coworkers point out a few clinical features that might help to differ between the two [3]. Abnormally high levels of lactate in proportion to the degree of hypoperfusion, which are not responding to adequate liquid and vasopressor therapy, is one of these. Other diagnostic clues might be high levels of lactate dehydrogenase (due to massive cell destruction) and hypoglycemia (due to impaired gluconeogenesis). Lactic acidosis in association with hematological malignancy is also a negative prognostic predictor - Glasheen and coworkers found a mortality rate of $>70 \%$ after one month in the case of lactic acidosis induced by Non-Hodgkin lymphoma [6].

The initial working hypothesis to this patient's extensive lactic acidosis was reduced peripheral circulation following cardiac arrest. The patient did not, however, respond biochemically to abundant liquid and vasopressor resuscitation, which could be expected if isolated hypoperfusion or hypoxia was the case (type A lactic acidosis). According to international guidelines for treatment of severe sepsis and septic shock [7], fluid resuscitation was performed by giving crystalloids only. The effect of catecholamines could in this case be partly reduced due to the severe and persistent acidosis $(\mathrm{pH}$ values below 7.2). Attempts to increase $\mathrm{pH}$ by using buffer, and thereby achieving effective catecholamines, did not succeed.

Another possible contributing factor to our patient's everincreasing concentration of lactate, might be the impairment of hepatic lactate clearance due to acute hypoxic liver injury (as 
indicated by slightly abnormal coagulation tests and elevated transaminase levels). It has been shown that patients with acute liver failure actually have a splanchnic release of lactate [8], thought to be due to accelerated splanchnic glycolysis and defects in hepatic pyruvate metabolism [9]. In healthy individuals, hepatic lactate clearance is estimated to be up to $70 \%$ of the whole body clearance. The rest is mainly cleared by the kidneys. Given the patient's anuric state after admission to the ICU, one might suspect acute hypoxic renal injury resulting from prolonged cardiopulmonary resuscitation - thus, further reducing the lactate clearance capacity.

Intracranial bleeding, a well-known complication shown to be more frequently occurring in association with AML than other hematological malignancies [10], was the cause of death in this case. Given the prolonged resuscitation time, one might suggest that the possibility of brain death should have been considered already at the time of admission to the ICU. This was, however, not the physicians' main focus given the dramatic clinical setting and rapid development of symptoms.

The detection of abnormally high levels of white blood cells and blood smear with immature cells at the time of arrival to the ICU, gave an early diagnostic clue indicating malignant disease of the blood. Other causes of type B lactic acidosis were less probable based on medical history and the list of medication. As this case illustrates, underlying hematological malignancy should be ruled out when facing persistent lactic acidosis after adequate systemic perfusion has been reestablished.

\section{References}

1. Cohen RD, W.H., Clinical and Biochemical Aspects of Lactate Acidosis. Blackwell Scientific Publications, Oxford, 1976.

2. Perrone J, Phillips C, Gaieski D (2011). Occult metformin toxicity in three patients with profound lactic acidosis. J Emerg Med 40: 271-5.

3. De Raes EA, Benoit DD, Depuydt PO, Offner F, Nollet J, et al. (2012). Early recognition of malignant lactic acidosis in clinical practice: report on 6 patients with haematological malignancies. Acta Clin Belg 67: 347-51.

4. De Keulenaer B, Van Outryve S, De Backer A, Van Overbeke L, Daelemans $\mathrm{R}$, et al. (2003). Symptomatic lactic acidosis due to relapse of T-cell acute lymphoblastic leukaemia in the kidney. Nephrol Dial Transplant 18: 1214-6.

5. Sillos EM, Shenep JL, Burghen GA, Pui CH, Behm FG et al. (2001). Lactic acidosis: a metabolic complication of hematologic malignancies: case report and review of the literature. Cancer 92: 2237-46.

6. Glasheen JJ, Sorensen MD (2005). Burkitt's lymphoma presenting with lactic acidosis and hypoglycemia---a case presentation. Leuk Lymphoma 46: 281-3.

7. Dellinger RP, Levy MM, Rhodes A, Annane D, Gerlach H, et al. (2013). Surviving sepsis campaign: international guidelines for management of severe sepsis and septic shock, 2012. Intensive Care Med 39: 165-228.

8. Murphy ND, Kodakat SK, Wendon JA, Jooste CA, Muiesan P, et al. (2001). Liver and intestinal lactate metabolism in patients with acute hepatic failure undergoing liver transplantation. Crit Care Med 29: 2111-8.

9. Jeppesen JB, Mortensen C, Bendtsen F, Moller S (2013). Lactate metabolism in chronic liver disease. Scand J Clin Lab Invest.

10. Chen CY, Tai CH, Cheng A, Wu HC, Tsay W et al. (2012). Intracranial hemorrhage in adult patients with hematological malignancies. BMC Med 10: 97. 